\title{
A CHECKLIST OF BIRDS OF NELLIAMPATHY HILLS, SOUTHERN WESTERN GHATS
}

\author{
J. Praveen ${ }^{1}$ and P.O. Nameer ${ }^{2}$
}

114/779(2), Ambadi, Kunnathurmedu P.O., Palakkad, Kerala 678013, India

${ }^{2}$ Assistant Professor, Department of Wildlife Sciences, College of Forestry, Kerala Agricultural University, Thrissur, Kerala 680656, India Email: ${ }^{1}$ paintedstork@gmail.com; ${ }^{2}$ nameer.ommer@gmail.com

\begin{abstract}
A bird study conducted at Nelliampathies, southern Western Ghats came up with a total checklist of 197 species with seven Red List and 13 Range Restricted species. A comprehensive checklist of 233 species, which includes observations from several previous studies, is provided. Range extension is reported in two subspecies, Grey-breasted Laughingthrush (Garrulax jerdoni fairbanki) and Brown Rock Pipit (Anthus similis travancoriensis) found in the southern Western Ghats. Moreover, 16 species of birds that were recorded by A.P. Kinloch in late 1920 s could not be located during the present study. In comparison with earlier studies by A.P. Kinloch, there is a general down trend in birds occupying the under storey vegetation which is of great concern.
\end{abstract}

\section{KEYwORDS}

Bird, checklist, endemic, Kerala, Nelliampathy, range extension, threatened, Western Ghats

The Western Ghats, identified as one of the biodiversity hotspots of the world, is a $1,600 \mathrm{~km}$ long chain of mountain ranges running parallel to the western coast of the Indian peninsula. The Palghat (Palakkad) gap is about $40 \mathrm{~km}$ wide lowland, which breaks this otherwise continuous mountain range. It forms a major natural barrier between the high hills of the Nilgiris and Anamalais, isolating the endemic life forms of the two high altitude habitats. Nelliampathy hills (or Nelliampathies) is a mountainous and plateau land lying immediately south of the Palghat gap. Biogeo-graphically, these forests are of great interest as they form the northern most range for many species of life forms that are endemic to southern Western Ghats

The Nelliampathy hills form the southern and the southwestern margin of the Palghat gap and they extend south, enclosing the Parambikulam basin. The western edge of the Nelliampathies gradually descends to the midlands of Thrissur district through a wide foothill zone drained by Puzhackal and Karuvannur rivers. The northwestern corner of the Nelliampathies extends far northwest almost up to Bharathapuzha as a 'spur hill', the Pattikkad-VadakkancherryVazhani hills. The Parambikulam basin drains towards south and west through the Chalakkudy river. The eastern border of the Parambikulam basin so formed by the Anamalais, a high ridge which starts from near the southeastern corner of the Palghat gap and sweeps south and southeast meeting with the High Range, near Anamudi. South, in between the southern Idamalar-Pooyamkutty valley, and the southern edge of the Parambikulam basin, there is the east-west Chalakkudy valley, the upper reaches of which are formed by Sholayar, a tributary of the Chalakkudy river and the lower reaches by the main Chalakkudy river. Part of the Anamalais, upper reaches of Sholayar and the eastern parts of the Parambikulam basin are in Tamil Nadu. This extensive, slightly lower basin, sheltered by the Nelliampathies in the north, Anamalais in the east and the Kannan Devan hill in the south draining through the Periyar as well as the Chalakkudy rivers had, until recently, one of the most extensive tropical moist forests in the entire Western Ghats (Nair, 1991).

The present study attempts to assess the avifaunal wealth of Nelliampathy hills, which has been considered as quite rich and diverse. A.P. Kinloch, an early time British planter, had reported a series of observations from these hills during the early $20^{\text {th }}$ century (Kinloch $\left.1921 \mathrm{a}, \mathrm{b}, \mathrm{c} ; 1923 \mathrm{a}, \mathrm{b}\right)$. Since then, apart from random reports and observations (Hussain \& Khan, 1977; Khan, 1977; Vijayan, 1978; Zacharias \& Gaston, 1999; Prasad \& Vijayan, 2002; Praveen (unpublished data from 1996 to 2003)), there has not been any comprehensive study in these hills. In this context, this work comes at the right time where we also try to compare in qualitative terms the changes in ornithology in these hills, in comparison with the land use changes, from the times of Kinloch.

\section{Study Area}

The study area lies between the geographical coordinates of $10^{\circ} 20^{\prime}-10^{\circ} 48^{\prime} \mathrm{N} \& 76^{\circ} 30^{\prime}-76^{\circ} 55^{\prime} \mathrm{E}$, forming part of Anamalai - Palani hill block in the southern Western Ghats. The region falls under administrative boundary of Chittur taluk in Palakkad district, Kerala. The forest zone comes under the Nemmara forest division consisting of two forest ranges, Nelliampathy range $\left(100 \mathrm{~km}^{2}\right)$ and Kollengode range $\left(60 \mathrm{~km}^{2}\right)$. Nelliampathies is bordered on the north by the Palghat gap, south by Parambikulam Wildlife Sanctuary, east by Indira Gandhi Wildlife Sanctuary, and west by Chalakudy forest division.

\section{Methodology}

The study was conducted for three months between December 2004 and February 2005. Two intensive surveys were carried out between these periods each having durations of five days each, where in about 40 birdwatchers from southern India participated. For the intensive survey seven base camps were selected within Nelliampathies. They are Kaikatty, Karimala, Lower Nelliampathies, Nellikkulam peak, Pakuthippalam, Pothumala and Pothundy dam. The base camps were selected in such a way that all the representative habitats of Nelliampathies were covered. At each camp four to six volunteers camped. Prefixed transects radiating from the base camps were followed in the morning (0730hr to 1100hr) and in the afternoon (1430hr to $1800 \mathrm{hr}$ ). While walking on the transects all the birds encountered were recorded species-wise.

Manuscript 1654; (C) ZOO; Date of publication 21 May 2007 Received 04 October 2006; Revised received 31 January 2007; Finally accepted 16 March 2007 
Table 1. Consolidated checklist of birds of Nelliampathies, along with the frequency of bird species sightings from different habitats

\begin{tabular}{|c|c|c|c|c|c|c|c|c|c|c|c|c|c|c|c|c|}
\hline & \multirow[t]{2}{*}{ Common Name } & \multirow[t]{2}{*}{ Scientific name } & \multicolumn{8}{|c|}{ Localities with sightings } & \multicolumn{3}{|c|}{$\begin{array}{l}\text { Past } \\
\text { records }\end{array}$} & \multicolumn{3}{|c|}{$\begin{array}{l}\text { Present } \\
\text { records \# }\end{array}$} \\
\hline & & & 1 & 2 & 3 & 4 & 5 & 6 & 7 & 8 & 9 & 10 & 11 & 12 & 13 & 14 \\
\hline 1. & Little Cormorant (28) & Phalacrocorax niger (Vieillot, 1817) & & & $x$ & & & & & $x$ & & & $x$ & 0 & 0 & 6 \\
\hline 2. & Great Cormorant (26) & Phalacrocorax carbo (Linnaeus, 1758) & & & & & & & & $\mathrm{X}$ & & & & 0 & 0 & 0 \\
\hline 3. & Little Egret (49) & Egretta garzetta (Linnaeus, 1766) & & & $\mathrm{x}$ & & & & & $\mathrm{X}$ & & & $x$ & 0 & 0 & 3 \\
\hline 4. & Large Egret (45-46) & Casmerodius albus (Linnaeus, 1758) & & & $x$ & & & & & $x$ & & & $\mathrm{X}$ & 0 & 0 & 1 \\
\hline 5. & Median Egret $(47,48)$ & Mesophoyx intermedia (Wagler, 1829) & & & $x$ & & & & & $\mathrm{X}$ & & & $x$ & 0 & 0 & 0 \\
\hline 6. & Cattle Egret (44) & Bubulcus ibis (Linnaeus, 1758) & & & $x$ & & & & & $x$ & & & $\mathrm{X}$ & 0 & 0 & 3 \\
\hline 7. & Indian Pond-Heron (42-42a) & Ardeola grayii (Sykes, 1832) & $x$ & & $x$ & $X$ & $x$ & & & $\mathrm{X}$ & & & $x$ & 1 & 0 & 9 \\
\hline 8. & Black-crowned Night-Heron (52) & Nycticorax nycticorax (Linnaeus, 1758) & & & & & & & & $\mathrm{X}$ & & & & 0 & 0 & 0 \\
\hline 9. & Cinnamon Bittern & Ixobrychus cinnamomeus (Gmelin, 1789) & & & & & & & & $\mathrm{X}$ & & & & 0 & 0 & 0 \\
\hline 10. & Jerdon's Baza (125-126) & Aviceda jerdoni (Blyth, 1842) & & & & & $x$ & & & & & & $\mathrm{X}$ & 1 & 0 & 0 \\
\hline 11. & Black Baza (127-128a) & Aviceda leuphotes (Dumont, 1820) & & & & $\mathrm{X}$ & & & & & & & $x$ & 1 & 0 & 0 \\
\hline 12. & Oriental Honey-Buzzard (129-130) & Pernis ptilorhynchus (Temminck, 1821) & & & & & $\mathrm{X}$ & $x$ & & $x$ & & & $X$ & 0 & 2 & 1 \\
\hline 13. & Black-shouldered Kite (124) & Elanus caeruleus (Desfontaines, 1789) & & & & & & & $x$ & $x$ & $x$ & & $\mathrm{X}$ & 0 & 0 & 2 \\
\hline 14. & Black Kite (132-134) & Milvus migrans (Boddaert, 1783) & & & & & & & & $\mathrm{X}$ & $\mathrm{X}$ & & & 0 & 0 & 0 \\
\hline 15. & Brahminy Kite (135) & Haliastur indus (Boddaert, 1783) & $\mathrm{X}$ & & $\mathrm{x}$ & & $\mathrm{X}$ & & & $\mathrm{X}$ & $\mathrm{X}$ & & $x$ & 1 & 0 & 5 \\
\hline 16. & Indian White-backed Vulture (185) & Gyps bengalensis (Gmelin, 1788) & & & & & & & & & $X$ & & & 0 & 0 & 0 \\
\hline 17. & Short-toed Snake-Eagle (195) & Circaetus gallicus (Gmelin, 1788) & $\mathrm{X}$ & & & & & & & & & & $\mathrm{X}$ & 0 & 0 & 0 \\
\hline 18. & ${ }^{*}$ Crested Serpent-Eagle (196-200) & Spilornis cheela (Latham, 1790) & $\mathrm{x}$ & $\mathrm{X}$ & & $x$ & $x$ & $x$ & $\mathrm{X}$ & $\mathrm{x}$ & $X$ & & $x$ & 6 & 1 & 4 \\
\hline 19. & Western Marsh-Harrier (193) & Circus aeruginosus (Linnaeus, 1758) & & & & & & & & $\mathrm{X}$ & & & & 0 & 0 & 0 \\
\hline 20. & Pallid Harrier & Circus macrourus (S.G. Gmelin, 1770) & & & & & & $\mathrm{X}$ & & & & & $x$ & 0 & 2 & 0 \\
\hline 21. & Crested Goshawk (144-146) & Accipiter trivirgatus (Temminck, 1824) & & & & $X$ & & & & & $X$ & & $X$ & 1 & 0 & 1 \\
\hline 22. & *Shikra $(137-140)$ & Accipiter badius (Gmelin, 1788) & & & & & & & & $x$ & $\mathrm{X}$ & & & 0 & 0 & 0 \\
\hline 23. & *Besra Sparrowhawk (149-151) & Accipiter virgatus (Temminck, 1822) & & $\mathrm{X}$ & & $\mathrm{X}$ & & & & $x$ & $X$ & & $x$ & 0 & 0 & 0 \\
\hline 24. & Common Buzzard (155-156) & Buteo buteo Linnaeus, 1758 & $\mathrm{X}$ & & & & & & & $\mathrm{X}$ & & & $x$ & 0 & 1 & 0 \\
\hline 25. & Black Eagle (172) & Ictinaetus malayensis (Temminck, 1822) & $x$ & $\mathrm{X}$ & & & $\mathrm{X}$ & & & $\mathrm{X}$ & $\mathrm{X}$ & & $x$ & 8 & 0 & 1 \\
\hline 26. & Booted Eagle (164) & Hieraaetus pennatus (Gmelin, 1788) & & $x$ & & $\mathrm{X}$ & & $\mathrm{X}$ & & $x$ & & & $x$ & 1 & 2 & 2 \\
\hline 27. & Rufous-bellied Eagle (165) & Hieraaetus kienerii (E. Geoffroy, 1835) & $\mathrm{X}$ & $\mathrm{X}$ & $\mathrm{X}$ & $X$ & $\mathrm{X}$ & & & $\mathrm{X}$ & & & $X$ & 1 & 0 & 4 \\
\hline 28. & Changeable Hawk-Eagle (160-162) & Spizaetus cirrhatus (Gmelin, 1788) & & & & $\mathrm{X}$ & $x$ & & & $\mathrm{X}$ & $X$ & & $x$ & 1 & 0 & 4 \\
\hline 29. & Common Kestrel (222-224) & Falco tinnunculus Linnaeus, 1758 & $\mathrm{X}$ & & & & & $\mathrm{X}$ & $x$ & $\mathrm{X}$ & $\mathrm{X}$ & & $x$ & 3 & 8 & 0 \\
\hline 30. & Oriental Hobby (214-215) & Falco severus Horsfield, 1821 & & & & & & $?$ & & & $\mathrm{X}$ & & & 0 & 0 & 0 \\
\hline 31. & Peregrine Falcon (209-211) & Falco peregrinus peregrinator & & & & & & & $x$ & & $X$ & & $x$ & 0 & 0 & 1 \\
\hline 32. & Grey Francolin (244-246) & Francolinus pondicerianus (Gmelin, 1789) & & & $\mathrm{x}$ & & & & & $\mathrm{X}$ & & & $x$ & 0 & 0 & 0 \\
\hline 33. & Jungle Bush-Quail (255-258) & Perdicula asiatica (Latham, 1790) & & & & & & $?$ & & & & & & 0 & 0 & 0 \\
\hline 34. & Painted Bush-Quail (262-263) & Perdicula erythrorhyncha (Sykes, 1832) & & & & & & & $\mathrm{X}$ & $\mathrm{X}$ & $\mathrm{X}$ & & $x$ & 0 & 1 & 0 \\
\hline 35. & Red Spurfowl (275-277) & Galloperdix spadicea (Gmelin, 1789) & $x$ & $\mathrm{X}$ & & & & & & $x$ & $X$ & & $x$ & 3 & 0 & 1 \\
\hline 36. & Grey Junglefowl (301) & Gallus sonneratii Temminck, 1813 & $x$ & $x$ & & $\mathrm{X}$ & $\mathrm{x}$ & $x$ & $\mathrm{X}$ & $x$ & $\mathrm{X}$ & & $x$ & 14 & 2 & 14 \\
\hline 37. & White-breasted Waterhen (343-345) & Amaurornis phoenicurus (Pennant, 1769) & $x$ & & & $\mathrm{X}$ & & & & $x$ & & & $x$ & 0 & 0 & 2 \\
\hline 38. & Bronze-winged Jacana (359) & Metopidius indicus (Latham, 1790) & & & & & & & & $\mathrm{X}$ & & & & 0 & 0 & 0 \\
\hline 39. & Little Ringed Plover (379-380) & Charadrius dubius Scopoli, 1786 & & & & & & & & $\mathrm{X}$ & & & & 0 & 0 & 0 \\
\hline 40. & Yellow-wattled Lapwing (370) & Vanellus malabaricus (Boddaert, 1783) & & & $\mathrm{X}$ & & & & & & & & $X$ & 0 & 0 & 2 \\
\hline 41. & Red-wattled Lapwing (366-368) & Vanellus indicus (Boddaert, 1783) & $x$ & & $x$ & & & & & $\mathrm{X}$ & & & $x$ & 0 & 0 & 6 \\
\hline 42. & Eurasian Woodcock (411) & Scolopax rusticola Linnaeus, 1758 & & & & & & & & & $X$ & & & 0 & 0 & 0 \\
\hline 43. & Common Greenshank (396) & Tringa nebularia (Gunner, 1767) & & & & & & & & & $X$ & & & 0 & 0 & 0 \\
\hline 44. & Green Sandpiper (397) & Tringa ochropus Linnaeus, 1758 & & & $x$ & & & & & $x$ & & & $x$ & 0 & 0 & 0 \\
\hline 45. & Common Sandpiper (401) & Actitis hypoleucos Linnaeus, 1758 & & & $x$ & & & & & $x$ & & & $x$ & 0 & 0 & 0 \\
\hline 46. & River Tern (463) & Sterna aurantia J.E. Gray, 1831 & & & $x$ & & & & & $x$ & & & $x$ & 0 & 0 & 0 \\
\hline 47. & Blue Rock Pigeon (516-517) & Columba livia Gmelin, 1789 & & & $x$ & & & & & $X$ & & & $x$ & 0 & 0 & 1 \\
\hline 48. & Nilgiri Wood-Pigeon (521) & Columba elphinstonii (Sykes, 1833) & & & & & & $x$ & $x$ & & & & $x$ & 3 & 1 & 0 \\
\hline 49. & Spotted Dove (537-540) & Streptopelia chinensis (Scopoli, 1786) & $\mathrm{X}$ & $\mathrm{X}$ & $x$ & & & & $x$ & $\mathrm{X}$ & $X$ & & $x$ & 1 & 0 & 11 \\
\hline 50. & Emerald Dove (542-544a) & Chalcophaps indica (Linnaeus, 1758) & $x$ & $x$ & & $\mathrm{X}$ & $\mathrm{X}$ & $\mathrm{X}$ & $x$ & $\mathrm{X}$ & $X$ & & $\mathrm{X}$ & 15 & 3 & 10 \\
\hline 51. & Pompadour Green-Pigeon (496-500) & Treron pompadora (Gmelin, 1789) & $\mathrm{X}$ & $\mathrm{X}$ & & $X$ & $\mathrm{X}$ & & $\mathrm{X}$ & $\mathrm{X}$ & $X$ & & $x$ & 15 & 0 & 27 \\
\hline 52. & Yellow-legged Green-Pigeon (503-505) & Treron phoenicoptera (Latham, 1790) & & & & & & & & $\mathrm{X}$ & & & & 0 & 0 & 0 \\
\hline 53. & Green Imperial-Pigeon & Ducula aenea (Linnaeus, 1766) & & & & & & & & $\mathrm{X}$ & & & & 0 & 0 & 0 \\
\hline 54. & Mountain Imperial-Pigeon (510-512) & Ducula badia (Raffles, 1822) & $x$ & & & $x$ & $x$ & $\mathrm{X}$ & $x$ & $x$ & $X$ & & $x$ & 28 & 1 & 40 \\
\hline 55. & Indian Hanging-Parrot $(566,567)$ & Loriculus vernalis (Sparrman, 1787) & $\mathrm{X}$ & $\mathrm{X}$ & & $\mathrm{X}$ & $x$ & $\mathrm{X}$ & & $\mathrm{X}$ & $X$ & & $x$ & 4 & 1 & 5 \\
\hline 56. & Rose-ringed Parakeet (549-550) & Psittacula krameri (Scopoli, 1769) & $?$ & & $\mathrm{x}$ & & $x$ & & & $x$ & & & $x$ & 4 & 0 & 2 \\
\hline 57. & Plum-headed Parakeet (557-558) & Psittacula cyanocephala (Linnaeus, 1766) & $x$ & $x$ & & & $x$ & $x$ & $x$ & $x$ & & & $x$ & 6 & 4 & 9 \\
\hline 58. & Blue-winged Parakeet (564) & Psittacula columboides (Vigors, 1830) & $x$ & & & $\mathrm{X}$ & $x$ & $x$ & $x$ & $\mathrm{X}$ & $x$ & & $x$ & 44 & 0 & 103 \\
\hline 59. & Red-winged Crested Cuckoo (569) & Clamator coromandus (Linnaeus, 1766) & & & & & $\mathrm{X}$ & & $\mathrm{x}$ & & & & $x$ & 2 & 0 & 0 \\
\hline 60. & Brainfever Bird (573-574) & Hierococcyx varius (Vahl, 1797) & & & & & & & $\mathrm{X}$ & $\mathrm{X}$ & $x$ & & $x$ & 0 & 0 & 1 \\
\hline 61. & Indian Cuckoo (576) & Cuculus micropterus Gould, 1838 & & & & & $x$ & & & & & & $\mathrm{X}$ & 0 & 0 & 1 \\
\hline 62. & Banded Bay Cuckoo (582-583) & Cacomantis sonneratii (Latham, 1790) & & & & & & & & $\mathrm{X}$ & & & & 0 & 0 & 0 \\
\hline 63. & Asian Koel (590-592) & Eudynamys scolopacea (Linnaeus, 1758) & & & & & & & & $x$ & & & & 0 & 0 & 0 \\
\hline 64. & ${ }^{*}$ Greater Coucal (600-602) & Centropus sinensis (Stephens, 1815) & $\mathrm{X}$ & $x$ & $x$ & & $x$ & & & $\mathrm{X}$ & $x$ & & $x$ & 1 & 0 & 0 \\
\hline 65. & Oriental Bay-Owl (609-610) & Phodilus badius (Horsfield, 1821) & & & & & & & & & & $\mathrm{x}$ & & 0 & 0 & 0 \\
\hline 66. & ${ }^{*}$ Oriental Scops-Owl (616-618b) & Otus sunia (Hodgson, 1836) & & & & $x$ & & & & $x$ & & & $x$ & 2 & 0 & 0 \\
\hline 67. & Collared Scops-Owl (619-624) & Otus bakkamoena Pennant, 1769 & & $x$ & & $x$ & & & & & & & $x$ & 0 & 0 & 0 \\
\hline 68. & Forest Eagle-Owl (628-629) & Bubo nipalensis Hodgson, 1836 & & & & & $x$ & & & & & & $x$ & 1 & 0 & 0 \\
\hline 69. & Brown Fish-Owl (631-632) & Ketupa zeylonensis (Gmelin, 1788) & & & & & $x$ & & & & & & $x$ & 1 & 0 & 0 \\
\hline 70. & Mottled Wood-Owl (655-657) & Strix ocellata (Lesson, 1839) & & & & & & & & $x$ & & & & 0 & 0 & 0 \\
\hline
\end{tabular}




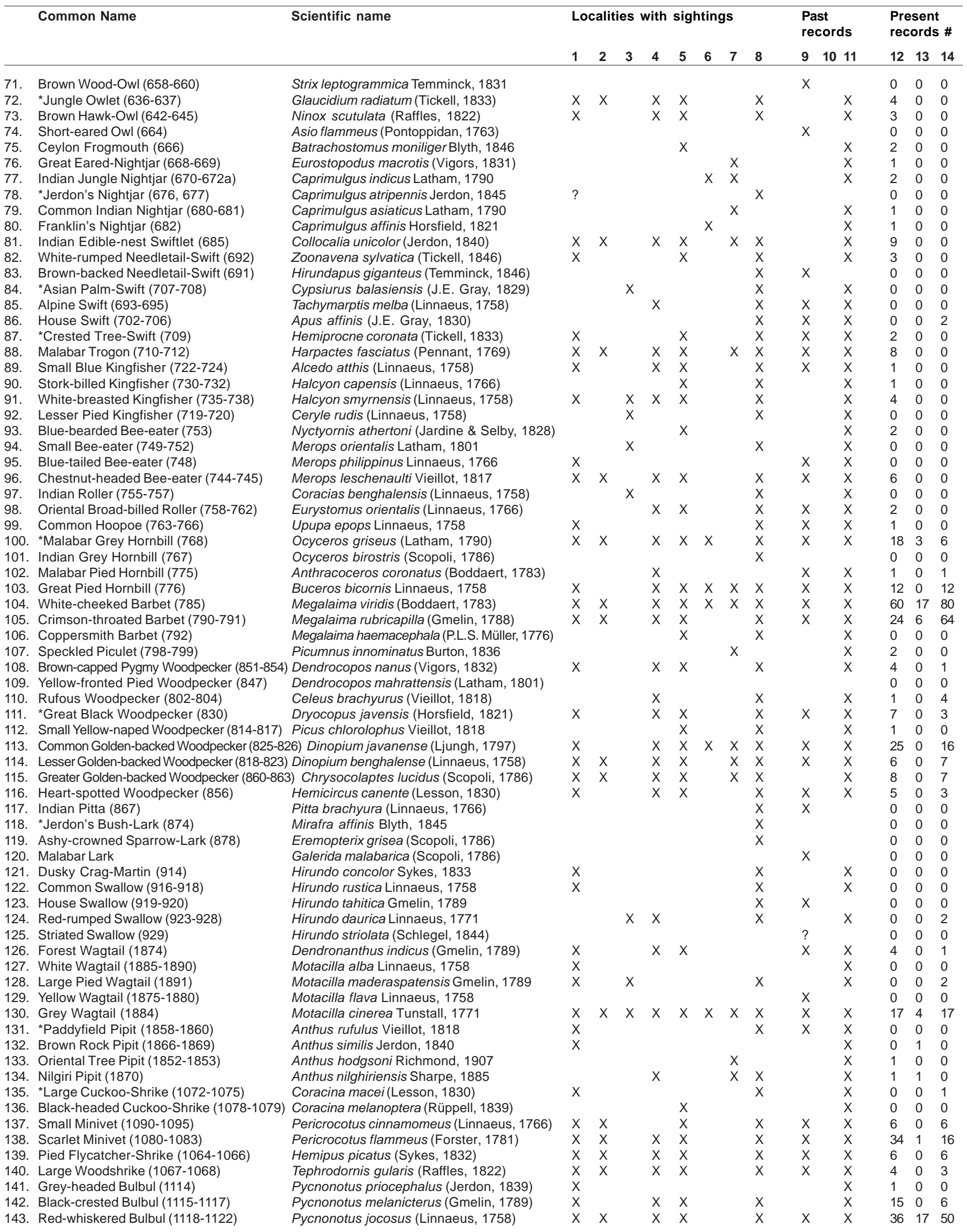




\begin{tabular}{|c|c|c|c|c|c|c|c|c|c|c|c|c|c|c|c|}
\hline & \multirow[t]{2}{*}{ Common Name } & \multirow[t]{2}{*}{ Scientific name } & \multicolumn{8}{|c|}{ Localities with sightings } & & $\begin{array}{l}\text { ast } \\
\text { cords }\end{array}$ & & $\begin{array}{l}\text { esen } \\
\text { cord }\end{array}$ & \\
\hline & & & 1 & 2 & 3 & 4 & 5 & 6 & 7 & 8 & 9 & 1011 & 12 & 13 & 14 \\
\hline 144. & Red-vented Bulbul (1126-1132) & Pycnonotus cafer (Linnaeus, 1766) & $\mathrm{x}$ & $\mathrm{x}$ & $\mathrm{x}$ & & & & $\mathrm{x}$ & $\mathrm{x}$ & & $\mathrm{x}$ & 5 & 0 & 7 \\
\hline 145. & Yellow-browed Bulbul (1143-1145) & Iole indica (Jerdon, 1839) & $\mathrm{x}$ & $\mathrm{x}$ & & $\mathrm{x}$ & $\mathrm{x}$ & $\mathrm{x}$ & $\mathrm{x}$ & $\mathrm{x}$ & $\mathrm{x}$ & $\mathrm{x}$ & 88 & 10 & 45 \\
\hline 146. & *Black Bulbul (1148-1151) & Hypsipetes leucocephalus (P.L.S. Muller, 1776) & $\mathrm{x}$ & & & $\mathrm{x}$ & $\mathrm{x}$ & $\mathrm{X}$ & $\mathrm{x}$ & $\mathrm{x}$ & $\mathrm{x}$ & $\mathrm{x}$ & 42 & 13 & 24 \\
\hline 147. & Common lora (1097-1101) & Aegithina tiphia (Linnaeus, 1758) & $\mathrm{x}$ & $\mathrm{x}$ & & & $\mathrm{x}$ & $\mathrm{x}$ & & $\mathrm{x}$ & & $\mathrm{x}$ & 10 & 1 & 1 \\
\hline 148. & Jerdon's Chloropsis (1107-1108) & Chloropsis cochinchinensis (Gmelin, 1788) & $\mathrm{x}$ & & & & $\mathrm{x}$ & & $\mathrm{X}$ & $\mathrm{X}$ & & $\mathrm{x}$ & 0 & 0 & 3 \\
\hline 149. & Gold-fronted Chloropsis (1103-1105) & Chloropsis aurifrons (Temminck, 1829) & $\mathrm{x}$ & $\mathrm{x}$ & & $\mathrm{x}$ & $x$ & & & $\mathrm{x}$ & $\mathrm{X}$ & $\mathrm{x}$ & 12 & 0 & 11 \\
\hline 150. & Asian Fairy-Bluebird $(1109,1110)$ & Irena puella (Latham, 1790) & $\mathrm{x}$ & $\mathrm{x}$ & & $\mathrm{x}$ & $\mathrm{x}$ & $\mathrm{x}$ & & $\mathrm{x}$ & $\mathrm{x}$ & $\mathrm{x}$ & 33 & 2 & 41 \\
\hline 151. & Brown Shrike (949-950a) & Lanius cristatus Linnaeus, 1758 & $\mathrm{x}$ & $\mathrm{x}$ & $\mathrm{x}$ & $\mathrm{x}$ & & & & $\mathrm{x}$ & & $\mathrm{x}$ & 5 & 0 & 6 \\
\hline 152. & Rufous-backed Shrike (946-948) & Lanius schach Linnaeus, 1758 & & & & & & & & & $\mathrm{x}$ & & 0 & 0 & 0 \\
\hline 153. & Blue-headed Rock-Thrush (1723) & Monticola cinclorhynchus (Vigors, 1832) & $\mathrm{x}$ & & & $\mathrm{x}$ & & $\mathrm{X}$ & & $\mathrm{X}$ & $\mathrm{X}$ & $\mathrm{x}$ & 1 & 1 & 1 \\
\hline 154. & Blue Rock-Thrush (1725-1726) & Monticola solitarius (Linnaeus, 1758) & & & & & & & & & $\mathrm{x}$ & & 0 & 0 & 0 \\
\hline 155. & Malabar Whistling-Thrush (1728) & Myiophonus horsfieldii (Vigors, 1831) & $\mathrm{x}$ & $\mathrm{x}$ & & $\mathrm{x}$ & $\mathrm{x}$ & $\mathrm{x}$ & $\mathrm{x}$ & $\mathrm{x}$ & $\mathrm{x}$ & $\mathrm{x}$ & 37 & 4 & 23 \\
\hline 156. & Pied Thrush (1731) & Zoothera wardii (Blyth, 1842) & & & & & & & & & $\mathrm{x}$ & & 0 & 0 & 0 \\
\hline 157. & Orange-headed Thrush (1734) & Zoothera citrina cyanotus (Jardine \& Selby, 1828) & $\mathrm{x}$ & $\mathrm{x}$ & & & & $\mathrm{x}$ & & $\mathrm{x}$ & $\mathrm{x}$ & $\mathrm{x}$ & 5 & 1 & 0 \\
\hline 158. & Scaly Thrush & Zoothera dauma (Latham, 1790) & & & & & & & & & $\mathrm{x}$ & & 0 & 0 & 0 \\
\hline 159. & Eurasian Blackbird (1751-1757) & Turdus merula Linnaeus, 1758 & $\mathrm{x}$ & & & & & & & $\mathrm{x}$ & $\mathrm{x}$ & $\mathrm{x}$ & 1 & 0 & 0 \\
\hline 160. & Indian Blue Robin $(1650,1651)$ & Luscinia brunnea (Hodgson, 1837) & & & & & & & & & $\mathrm{x}$ & & 0 & 0 & 0 \\
\hline 161. & Oriental Magpie-Robin (1661-166 & Copsychus saularis (Linnaeus, 1758) & $\mathrm{x}$ & $\mathrm{x}$ & $\mathrm{x}$ & $\mathrm{x}$ & $\mathrm{x}$ & & & $\mathrm{x}$ & & $\mathrm{x}$ & 0 & 0 & 6 \\
\hline 162. & Indian Robin (1717-1721) & Saxicoloides fulicata (Linnaeus, 1776) & & $\mathrm{x}$ & $\mathrm{x}$ & & & & & $\mathrm{x}$ & & $\mathrm{x}$ & 0 & 0 & 3 \\
\hline 163. & Pied Bushchat (1700-1703) & Saxicola caprata (Linnaeus, 1766) & $\mathrm{x}$ & & & & & & & $\mathrm{x}$ & & $\mathrm{x}$ & 0 & 0 & 1 \\
\hline 164. & *Wynaad Laughingthrush (1287) & Garrulax delesserti (Jerdon, 1 & $\mathrm{x}$ & $\mathrm{x}$ & & $\mathrm{x}$ & $\mathrm{x}$ & & & $\mathrm{x}$ & $\mathrm{x}$ & $\mathrm{x}$ & 8 & 0 & 4 \\
\hline 165. & Grey-breasted Laughingthrush (1309-1311) & Garrulax jerdoni Blyth, 1851 & & & & & & $\mathrm{x}$ & & & & $\mathrm{x}$ & 0 & 4 & 0 \\
\hline 166. & Spotted Babbler (1152-1159) & Pellorneum ruficeps Swainson & $\mathrm{x}$ & & & $\mathrm{x}$ & $\mathrm{x}$ & $\mathrm{X}$ & $\mathrm{x}$ & $\mathrm{x}$ & $\mathrm{x}$ & $\mathrm{x}$ & 6 & 2 & 4 \\
\hline 167. & *Indian Scimitar-Babbler (1172-1177) & Pomatorhinus horsfieldii Sykes, 1832 & $\mathrm{x}$ & $\mathrm{x}$ & & $\mathrm{x}$ & $\mathrm{x}$ & $\mathrm{x}$ & $\mathrm{x}$ & $\mathrm{x}$ & $\mathrm{x}$ & $\mathrm{x}$ & 20 & 1 & 3 \\
\hline 168. & Rufous-bellied Babbler (1219-1223) & Dumetia hyperythra (Franklin, 1831) & & & & & & & $\mathrm{x}$ & $x$ & & $\mathrm{x}$ & 0 & 0 & 0 \\
\hline 169. & Black-headed Babbler (1224-1227) & Rhopocichla atriceps (Jerdon, 1839) & $\mathrm{x}$ & $\mathrm{x}$ & & $\mathrm{x}$ & $\mathrm{x}$ & & & $\mathrm{x}$ & $\mathrm{X}$ & $\mathrm{x}$ & 17 & 0 & 3 \\
\hline 170. & Indian Rufous Babbler (1259-1260) & Turdoides subrufus (Jerdon, 1839) & $x$ & & & $x$ & $\mathrm{x}$ & & & $\mathrm{x}$ & & $\mathrm{x}$ & 3 & 0 & 1 \\
\hline 171. & Jungle Babbler (1261-1265) & Turdoides striatus (Dumont, 1823) & & $\mathrm{x}$ & & $\mathrm{x}$ & $\mathrm{x}$ & & & $\mathrm{x}$ & & $\mathrm{x}$ & 2 & 0 & 9 \\
\hline 172. & White-headed Babbler (1267-1268) & Turdoides affinis (Jerdon, 1847) & & & $\mathrm{x}$ & & & & & $\mathrm{x}$ & & $\mathrm{x}$ & 0 & 0 & 1 \\
\hline 173. & Quaker Tit-Babbler (1389-1391) & Alcippe poioicephala (Jerdon, 1844) & $x$ & $\mathrm{x}$ & & $x$ & $\mathrm{x}$ & $\mathrm{x}$ & $\mathrm{x}$ & $\mathrm{x}$ & & $\mathrm{x}$ & 28 & 3 & 16 \\
\hline 174. & Streaked Fantail-Warbler (1498- & Cisticola juncidis (Rafinesque, 1810) & & & & & & & & $x$ & & & 0 & 0 & 0 \\
\hline 175. & Franklin’s Prinia (1502-1505) & Prinia hodgsonii Blyth, 1844 & $\mathrm{x}$ & & & & & $x$ & & $\mathrm{x}$ & & $\mathrm{x}$ & 3 & 3 & 0 \\
\hline 176. & Ashy Prinia (1515-1518) & Prinia socialis Sykes, 1832 & & & & & & & $\mathrm{x}$ & $\mathrm{x}$ & & $\mathrm{x}$ & 0 & 0 & 1 \\
\hline 177. & *Plain Prinia (1510-1514) & 7ornata Syk & & & $\mathrm{x}$ & & & & & $\mathrm{x}$ & & $\mathrm{x}$ & 0 & 0 & 1 \\
\hline 178. & Blyth's Reed-Warbler (1556) & ohalus dumetor & $\mathrm{x}$ & $\mathrm{x}$ & $\mathrm{x}$ & $\mathrm{x}$ & $\mathrm{X}$ & $\mathrm{X}$ & & $\mathrm{x}$ & & $\mathrm{x}$ & 9 & 5 & 18 \\
\hline 179. & Thick-billed Warbler (1549) & phalus aedon (Pall & & & & & & & & $\mathrm{x}$ & & & 0 & 0 & 0 \\
\hline 180. & Common Tailorbird (1535-1539) & Orthotomus sutorius (Pennant, 1769) & $\mathrm{x}$ & $\mathrm{x}$ & $x$ & & $\mathrm{x}$ & & & $\mathrm{x}$ & $\mathrm{x}$ & $\mathrm{x}$ & 3 & 0 & 1 \\
\hline 181. & *Tickell's Warbler (1579) & Phylloscopus affinis (Tickell, 1833) & & & & $\mathrm{x}$ & & & $x$ & & & $\mathrm{x}$ & 2 & 0 & 0 \\
\hline 182. & Greenish Leaf-Warbler ( 160 & Phylloscopus trochiloides (Sundeva & $x$ & $\mathrm{x}$ & & $\mathrm{x}$ & $\mathrm{x}$ & $\mathrm{x}$ & $x$ & $x$ & & $\mathrm{x}$ & 43 & 4 & 42 \\
\hline 183. & Large-billed Leaf-Warbler (1601) & Phylloscopus magnir & $\mathrm{x}$ & $\mathrm{x}$ & & $\mathrm{x}$ & $\mathrm{x}$ & $\mathrm{X}$ & $\mathrm{x}$ & $\mathrm{x}$ & & $\mathrm{x}$ & 31 & 2 & 22 \\
\hline 184. & *Western Crowned Warbler (1606) & Phylloscopus occipitalis (Blyth, 1845) & $\mathrm{x}$ & & & $\mathrm{x}$ & & $\mathrm{x}$ & & & & $\mathrm{x}$ & 1 & 4 & 0 \\
\hline 185. & Broad-tailed Grass-Warbler (1546) & Schoenicola platyura (Jerdon, 1844) & & & & & & & & $\mathrm{x}$ & & & 0 & 0 & 0 \\
\hline 186. & Asian Brown Flycatcher (1407) & Musc & $\mathrm{x}$ & $\mathrm{x}$ & & $\mathrm{x}$ & $\mathrm{x}$ & & $\mathrm{x}$ & $\mathrm{X}$ & $\mathrm{x}$ & $\mathrm{x}$ & 9 & 0 & 8 \\
\hline 187. & Rusty-tailed Flycatcher (1409) & ruficauda Swains & $\mathrm{x}$ & $\mathrm{x}$ & & & & & & $\mathrm{x}$ & $\mathrm{x}$ & $\mathrm{x}$ & 3 & 0 & 0 \\
\hline 188. & Brown-breasted Flycatcher (1408) & Muscic & $\mathrm{x}$ & $\mathrm{x}$ & & $\mathrm{x}$ & & $\mathrm{x}$ & & $\mathrm{x}$ & $\mathrm{x}$ & $\mathrm{x}$ & 2 & 2 & 1 \\
\hline 189. & Verditer Flycatcher (1445) & s thalassina (Swainson, 1838) & $\mathrm{x}$ & & & $\mathrm{x}$ & & $\mathrm{x}$ & $x$ & & $\mathrm{x}$ & $\mathrm{x}$ & 4 & 0 & 5 \\
\hline 190. & Nilgiri Flycatcher (1446) & Eumyias albicaudata (Jerdon, 1840) & & & & $\mathrm{x}$ & $\mathrm{x}$ & & $x$ & & $\mathrm{x}$ & $\mathrm{x}$ & 7 & 0 & 1 \\
\hline 191. & White-bellied Blue-Flycatcher (14 & Cyornis pallipes (Jerdon, 1840) & $\mathrm{x}$ & & & & $\mathrm{x}$ & & & & & $\mathrm{x}$ & 3 & 0 & 0 \\
\hline 192. & Blue-throated Flycatcher (1440) & Cyornis rubeculoides & & $\mathrm{x}$ & & & & & & $\mathrm{x}$ & & $\mathrm{x}$ & 1 & 0 & 0 \\
\hline 193. & Tickell's Blue-Flycatcher (1442-1 & Cyornis tickelliae $\mathrm{Bl}$ & & $\mathrm{x}$ & & & $\mathrm{x}$ & & & $\mathrm{x}$ & & $\mathrm{x}$ & 1 & 0 & 0 \\
\hline 194. & Grey-headed Flycatch & Culicica & & & & $\mathrm{x}$ & $\mathrm{x}$ & & $\mathrm{x}$ & $\mathrm{x}$ & $\mathrm{x}$ & $\mathrm{x}$ & 20 & 0 & 4 \\
\hline 195. & Asian Paradise-Flycatcher (1460-1464) & e paradisi (Linnael & $\mathrm{x}$ & $\mathrm{x}$ & & $\mathrm{x}$ & $\mathrm{x}$ & & & $\mathrm{x}$ & $\mathrm{x}$ & $\mathrm{x}$ & 12 & 0 & 5 \\
\hline 196. & Black-naped Monarch-Flycatcher (1465-1469) & Hypothymis azurea (Boddaert, 1783) & $\mathrm{x}$ & $\mathrm{x}$ & & $\mathrm{x}$ & $\mathrm{x}$ & & & $\mathrm{x}$ & & $\mathrm{x}$ & 10 & 0 & 3 \\
\hline 197. & Great Tit (1790-1797) & Parus major Linnaeus, 1758 & $\mathrm{x}$ & $\mathrm{x}$ & & $\mathrm{x}$ & $\mathrm{x}$ & $\mathrm{x}$ & $\mathrm{x}$ & $\mathrm{x}$ & $\mathrm{x}$ & $\mathrm{x}$ & 3 & 0 & 5 \\
\hline 198. & Black-lored Yellow Tit ( & Paru & & & & $\mathrm{x}$ & $\mathrm{X}$ & & $x$ & $\mathrm{x}$ & $\mathrm{x}$ & $\mathrm{x}$ & 9 & 0 & 5 \\
\hline 199. & Velvet & Sitta & $\mathrm{x}$ & $\mathrm{x}$ & & $\mathrm{x}$ & $\mathrm{x}$ & $\mathrm{x}$ & $x$ & $\mathrm{x}$ & $\mathrm{x}$ & $\mathrm{x}$ & 20 & 1 & 17 \\
\hline 200. & Thick-billed Flowerpecker (1892-1894) & Dicaeum agile (Tickell, 1833) & $\mathrm{x}$ & $\mathrm{x}$ & & & & & $x$ & $\mathrm{x}$ & & $\mathrm{x}$ & 7 & 0 & 5 \\
\hline 201. & Tickel & Dicat & & & & & & $\mathrm{x}$ & & $\mathrm{x}$ & & $\mathrm{x}$ & 2 & 3 & 9 \\
\hline 202. & Plain Flowerpecker (1901-1903) & concolor Jerdon, 1840 & $\mathrm{x}$ & $\mathrm{x}$ & & $\mathrm{x}$ & $\mathrm{x}$ & $x$ & & $\mathrm{x}$ & & $\mathrm{x}$ & 11 & 1 & 9 \\
\hline 203. & Sunbird (1907-1908) & Necta & & & $\mathrm{x}$ & ? & & $\mathrm{X}$ & $\mathrm{x}$ & $\mathrm{x}$ & $\mathrm{X}$ & $\mathrm{x}$ & 6 & 0 & 1 \\
\hline 204. & Small Sunbird (1909) & Necta & $\mathrm{x}$ & $\mathrm{x}$ & & $\mathrm{x}$ & $\mathrm{x}$ & $\mathrm{x}$ & $\mathrm{x}$ & $\mathrm{x}$ & & $\mathrm{x}$ & 47 & 6 & 33 \\
\hline 205. & Purple Sunbird (1916-1918) & Nectarinia asiatica (Latham, 1790) & $\mathrm{x}$ & $\mathrm{x}$ & & & & $\mathrm{x}$ & & $\mathrm{x}$ & & $\mathrm{x}$ & 5 & 0 & 6 \\
\hline 206. & Loten's Sunbird (1911-1912) & Nectarinia lotenia (Linnaeus, 1766) & $\mathrm{x}$ & $\mathrm{x}$ & & $\mathrm{x}$ & $\mathrm{x}$ & & & $\mathrm{x}$ & & $\mathrm{x}$ & 0 & 0 & 2 \\
\hline 207. & Little Spiderhunter (1931) & Arachnothera longirostra (La & $\mathrm{x}$ & & & $\mathrm{x}$ & $\mathrm{x}$ & $\mathrm{x}$ & & $\mathrm{x}$ & $\mathrm{x}$ & $\mathrm{x}$ & 9 & 0 & 8 \\
\hline 208. & Oriental White-eye (1933-1936) & Zosterops palpebrosus (Temminck, 1824) & $\mathrm{x}$ & & & $\mathrm{x}$ & $\mathrm{x}$ & $\mathrm{X}$ & & $\mathrm{x}$ & $\mathrm{x}$ & $\mathrm{x}$ & 28 & 5 & 6 \\
\hline 209. & n Rosefinch (2010-2013) & Carpodacu & $\mathrm{x}$ & & & $\mathrm{x}$ & & $\mathrm{x}$ & $\mathrm{x}$ & & $\mathrm{x}$ & $\mathrm{x}$ & 1 & 0 & 3 \\
\hline 210. & White-rumped Munia (1967-1970) & Lonchura striata (Linnaeus, 1766) & & & & & & & & $\mathrm{x}$ & & & 0 & 0 & 0 \\
\hline 211. & Black-throated Munia (1971-1973) & Lonchura kelaarti (Jerdon, 1863) & $\hat{\lambda}$ & & & $\mathrm{x}$ & $\mathrm{x}$ & & $x$ & $\mathrm{x}$ & $\mathrm{x}$ & $\mathrm{x}$ & 3 & 0 & 1 \\
\hline 212. & parrow (1938-1939a) & Passer domesticus (Linnaeus, 1758) & & & & & & & & $\mathrm{x}$ & & & 0 & 0 & 0 \\
\hline 213. & ed Sparrow (1948- & Petronia xanthocollis (Burton, 1838) & & & & & & & & $\mathrm{x}$ & & & 1 & 0 & 0 \\
\hline 214. & & Ploceus philippinus (Linnaeus, 1766) & & & & & & & & $\mathrm{x}$ & & & 0 & 0 & 0 \\
\hline 215. & Grey-headed Starling (987-989) & Sturnus malabaricus (Gmelin, 1789) & $x$ & & $\mathrm{x}$ & $\mathrm{x}$ & $\mathrm{x}$ & & & $\mathrm{x}$ & & $\mathrm{x}$ & 0 & 0 & 8 \\
\hline 216. & Grey-headed Starling (988) & Sturnus malabaricus blythi (Jerdon, 1844) & $\mathrm{x}$ & & $\mathrm{x}$ & $\mathrm{x}$ & $\mathrm{x}$ & & & & & $\mathrm{x}$ & 1 & 0 & 16 \\
\hline
\end{tabular}




\begin{tabular}{|c|c|c|c|c|c|c|c|c|c|c|c|c|c|c|c|c|}
\hline & \multirow[t]{2}{*}{ Common Name } & \multirow[t]{2}{*}{ Scientific name } & \multicolumn{8}{|c|}{ Localities with sightings } & \multicolumn{3}{|c|}{$\begin{array}{l}\text { Past } \\
\text { records }\end{array}$} & \multicolumn{3}{|c|}{$\begin{array}{l}\text { Present } \\
\text { records \# }\end{array}$} \\
\hline & & & 1 & 2 & 3 & 4 & 5 & 6 & 7 & 8 & 9 & 10 & 11 & 12 & 13 & 14 \\
\hline 217. & Brahminy Starling (994) & Sturnus pagodarum (Gmelin, 1789) & & & $\mathrm{x}$ & & & & & & & & $\mathrm{X}$ & 0 & 0 & 1 \\
\hline 218. & Common Myna (1006-1007) & Acridotheres tristis (Linnaeus, 1766) & $\mathrm{X}$ & & $x$ & $\mathrm{X}$ & & & $\mathrm{X}$ & $\mathrm{X}$ & & & $x$ & 0 & 0 & 12 \\
\hline 219. & Jungle Myna (1009-1011) & Acridotheres fuscus (Wagler, 1827) & & & & & $x$ & & & & & & $x$ & 0 & 0 & 0 \\
\hline 220. & *Southern Hill-Myna (1016) & Gracula indica (Cuvier, 1829) & $\mathrm{X}$ & & & $\mathrm{X}$ & $x$ & $\mathrm{x}$ & $x$ & $x$ & $\mathrm{X}$ & & $\mathrm{x}$ & 54 & 0 & 92 \\
\hline 221. & Eurasian Golden Oriole (952-953) & Oriolus oriolus (Linnaeus, 1758) & $\mathrm{X}$ & $x$ & & $X$ & $\mathrm{X}$ & $\mathrm{X}$ & $x$ & $x$ & $\mathrm{X}$ & & $\mathrm{X}$ & 11 & 2 & 27 \\
\hline 222. & *Black-naped Oriole $(954,956-957)$ & Oriolus chinensis Linnaeus, 1766 & & $\mathrm{x}$ & & & & & & & & & $\mathrm{X}$ & 0 & 0 & 0 \\
\hline 223. & Black-headed Oriole (958-960a) & Oriolus xanthornus (Linnaeus, 1758) & & $x$ & & $\mathrm{X}$ & & & & $x$ & & & $\mathrm{X}$ & 3 & 0 & 0 \\
\hline 224. & *Black Drongo (962-964) & Dicrurus macrocercus Vieillot, 1817 & & & $\mathrm{X}$ & & & & & $x$ & & & $\mathrm{X}$ & 0 & 0 & 1 \\
\hline 225. & Ashy Drongo (965-966b) & Dicrurus leucophaeus Vieillot, 1817 & $\mathrm{X}$ & $x$ & & $x$ & $\mathrm{X}$ & $x$ & $x$ & $\mathrm{X}$ & $\mathrm{X}$ & & $\mathrm{X}$ & 38 & 0 & 62 \\
\hline 226. & Bronzed Drongo (971) & Dicrurus aeneus Vieillot, 1817 & $\mathrm{X}$ & $\mathrm{X}$ & & $\mathrm{X}$ & $\mathrm{X}$ & & & $\mathrm{X}$ & $\mathrm{X}$ & & $\mathrm{X}$ & 6 & 0 & 12 \\
\hline 227. & Spangled Drongo (973) & Dicrurus hottentottus (Linnaeus, 1766) & & & & & $x$ & & & & & & $x$ & 2 & 0 & 0 \\
\hline 228. & Greater Racket-tailed Drongo (976-981) & Dicrurus paradiseus (Linnaeus, 1766) & $\mathrm{X}$ & $x$ & & $x$ & $x$ & & & $x$ & $\mathrm{X}$ & & $\mathrm{X}$ & 25 & 1 & 28 \\
\hline 229. & Ashy Woodswallow (982) & Artamus fuscus Vieillot, 1817 & & & $x$ & & & & & $\mathrm{x}$ & & & $\mathrm{x}$ & 0 & 0 & 0 \\
\hline 230. & Indian Treepie (1030a-1034) & Dendrocitta vagabunda (Latham, 1790) & & $\mathrm{X}$ & & & & & & $x$ & $\mathrm{X}$ & & $x$ & 2 & 0 & 1 \\
\hline 231. & White-bellied Treepie (1036) & Dendrocitta leucogastra Gould, 1833 & $\mathrm{X}$ & & & $x$ & $x$ & & $\mathrm{X}$ & $\mathrm{x}$ & $\mathrm{X}$ & & $x$ & 22 & 0 & 11 \\
\hline 232. & House Crow (1048-1051) & Corvus splendens Vieillot, 1817 & & & $x$ & & $x$ & & & $x$ & & & $x$ & 0 & 0 & 6 \\
\hline 233. & Jungle Crow (1054-1057) & Corvus macrorhynchos Wagler, 1827 & & & & $x$ & $\mathrm{X}$ & & $\mathrm{X}$ & $x$ & & & $\mathrm{X}$ & 2 & 0 & 6 \\
\hline
\end{tabular}

Legend: 1 - Kaikatty; 2 - Lower Nelliampathies; 3 - Pothundy Dam; 4 - Pothumala; 5 - Pakuthipalam; 6 - Nellikulam peak; 7 - Karimala; 8 - all sites together; 9 - J. Praveen pers. observ. between 1996 \& 2003; 10 - Kinloch (1921a,b,c \& 1923 a \& b); 11 - other published records; 12 - evergreen and moist deciduous forests; 13 - shola forests; 14 - plantation \& reservoir; \# - Present records with frequency of sightings from different habits Taxonomy and nomenclature follow Manakadan \& Pittie (2001)

Apart from this the number of individuals per species also was counted. This was continued for first two hours and then only those species that were seen on that transect, on that day alone were recorded.

All the birds opportunistically sighted/heard were recorded separately. This information was mainly for the preparation of the checklist. The birds were identified using binoculars (10x50 or 8x40) and field guides of Ali \& Ripley (1987); Grimmett et al. (1998); Kazmierczak (2000). The data thus collected were recorded in to the prescribed data sheet. Apart from the checklist the frequency of sighting of the birds in different habitats, such as the birds of the forests (evergreen and moist deciduous), birds of the shola forests and the birds of the plantations (cardamom, coffee, tea and orange), were also worked out and the results are presented.

\section{Results}

The present study of the avifauna of the Nelliampathy hills reported a total checklist of 197 species belonging to 50 families. Thus the total checklist of birds from Nelliampathy hills comes to 233 species in 64 families (Table 1). Also given in the Table 1 is the frequency of bird sightings from different major habitats at Nelliampathies. The earlier bird records from Nelliampathies are also given in the Table.

The plantations recorded higher number of species when compared to the shola forests. Daniels (1989) \& Daniels et al. (1990) also observed greater bird species diversity in secondary forest in Uttara Kannada district, Western Ghats. The high altitude areas are known to support fewer number of bird species when compared to the lower altitude areas. Studies on the birds of neotropics (Stotz et al., 1996) had shown that a plateau in species number between $500-1000 \mathrm{~m}$, then there is a decline in species richness as the altitude increases at the rate of approximately $5 \%$ with every $100 \mathrm{~m}$ gain in elevation from 1000-3000m. This further explains the lower species richness observed in the present study in the shola forest at higher reaches in Nelliampathies. Though the plantations recorded higher number of species than the natural forests, the individual bird species frequency analysis shows that the keystone indicator species prefer natural habitats over plantations.

Seven Red List book species (after Birdlife International, 2001) (Table 2) were recorded from Nelliampathies, while 13 species endemic to Western Ghats (after Stattersfield et al. 1998), (Table 3) were also noted. It is interesting to note that five out of the seven threatened species of birds recorded from Nelliampathies were absent in the plantations. In the case of endemic species of birds six species of Western Ghats endemics reported from the Nelliampathies could not be sighted from the plantations.

\section{Significant observations}

The following are the significant observations during the study.

Jerdon's Baza (Aviceda jerdoni): A single bird was seen at Pakuthippalam on 28 November 2003 during the pilot survey by Jafer Palot. Considered extremely rare in the Western Ghats, the species was not recorded by A.P. Kinloch during his studies in Nelliampathies.

Black Baza (Aviceda leuphotes): A single bird recorded from Pothumala on 28 December 2003 by P.O. Nameer and C. Sashikumar.

Short-toed Snake-Eagle (Circaetus gallicus): A single bird seen near Kaikatty by C. Sashikumar and P.O. Nameer during the pilot survey.

Nilgiri Wood-Pigeon (Columba elphinstonii): This endemic pigeon was regularly observed at Karimala during the pilot survey and also during the intensive survey period. A single bird was also recorded near Nellikkulam Peak during the pilot survey.

Red-winged Crested Cuckoo (Clamator coromandus): Two sightings of this cuckoo. One bird was seen at Pakuthippalam on 28 December 2003 by K.G. Raghu and one sighting from 
Table 2. The frequency of occurrence of threatened species of birds in natural forest and plantations at Nelliampathies, southern Western Ghats

\begin{tabular}{|c|c|c|c|}
\hline No.Species & IUCN Category & Natural Forest & Plantations \\
\hline 1. Great Pied Hornbill & Near Threatened & 26 & 16 \\
\hline 2. Nilgiri Flycatcher & Near Threatened & 7 & 2 \\
\hline 3. Nilgiri Wood-Pigeon & Vulnerable & 4 & 0 \\
\hline $\begin{array}{l}\text { 4. Grey-breasted } \\
\text { Laughingthrush }\end{array}$ & Near Threatened & 4 & 0 \\
\hline 5. Malabar Pied Hornbill & Near Threatened & 3 & 0 \\
\hline 6. Nilgiri Pipit & Near Threatened & 3 & 0 \\
\hline 7. Pallid Harrier & Near Threatened & 1 & 0 \\
\hline
\end{tabular}

Table 3. The frequency of occurrence of Western Ghats endemic species of birds in natural forest and plantations at Nelliampathies, southern Western Ghats

\begin{tabular}{|c|c|c|c|}
\hline No. & Species & $\begin{array}{l}\text { Natural forest } \\
\text { Freque }\end{array}$ & $\begin{array}{l}\text { Plantations } \\
\text { ency }\end{array}$ \\
\hline 1. & Blue-winged Parakeet & 103 & 257 \\
\hline 2. & Small Sunbird & 89 & 56 \\
\hline 3. & White-bellied Treepie & 37 & 10 \\
\hline 4. & Malabar Grey Hornbill & 34 & 9 \\
\hline 5. & Wynaad Laughingthrush & 30 & 10 \\
\hline 6. & Rufous Babbler & 8 & 6 \\
\hline 7. & Nilgiri Flycatcher & 7 & 2 \\
\hline 8. & Nilgiri Wood-Pigeon & 4 & 0 \\
\hline 9. & Grey-breasted Laughingthrush & 4 & 0 \\
\hline 10. & White-bellied Blue Flycatcher & 4 & 0 \\
\hline 11. & Grey-headed Bulbul & 3 & 0 \\
\hline 12. & Nilgiri Pipit & 3 & 0 \\
\hline
\end{tabular}

Karimala on 11 November 2004 by P.O. Nameer and E. Kunhikrishnan.

Ceylon Frogmouth (Batrachostomus moniliger): The bird was not recorded from any locality during the pilot survey period but two birds responded to tape recorded calls played by J. Praveen and P.P. Sreenivasan at Pakuthippalam during the intensive study phase. This species was not reported by A.P. Kinloch but probably got missed out because of its secretive diurnal habits.

Great-eared Nightjar (Eurostopodus macrotis): Not recorded from any of the localities except from Karimala where its calls were noted by Dipu Karuthedathu during the pilot survey period. A.P. Kinloch did not come across this bird in Nelliampathies during his stay.

Oriental Broad-billed Roller (Eurystomus orientalis): The bird was observed at Pakuthippalam during the pilot survey and intensive survey periods. It has been regularly recorded from here since 1996 (J. Praveen, pers. obser.; L. Namassivayan, pers. comm.; Bashir, pers. comm.).

Malabar Pied Hornbill (Anthracoceros coronatus): Two birds were observed in Pothumala on 27 and 28 December 2003 by P.O. Nameer \& C. Sashikumar near a coffee plantation. The bird is decidedly rare everywhere in Kerala and Pothumala adjoins the Vazhachal forests on the west, which is considered as one of the strongholds for this species. Kinloch (1921b,c; 1923a,b) noted this species as common in Nelliampathies but this is no longer true.

Great Pied Hornbill (Buceros bicornis): This bird got recorded from all the base camps during all visits.
Nelliampathies together with Parambikulam Wildlife Sanctuary houses one of the most viable populations of Great Hornbill in the entire Western Ghats.

Speckled Piculet (Picumnus innominatus): Two sightings of this bird from Karimala during the pilot survey period by Dipu Karuthedathu and P.P. Sreenivasan.

Great Black Woodpecker (Dryocopus javensis): Recorded regularly from the forests of Pakuthippalam, Pothumala and Kaikatty.

Grey-breasted Laughingthrush (Garrulax jerdoni fairbanki): P.K. Ravindran sight recorded this species on three occasions in a shola near Nellikkulam Peak (c. 1500m) on 28 December 2003 during the pilot survey. P.K. Ravindran \& J. Praveen recorded the bird calling from a shola once near Minnampara Peak (c. 1300m) on 12 November 2004 during an intensive study survey. Subspecies identification (of the birds seen near Nellikkulam Peak) is certain as of race fairbanki, observers clearly noted the lack of black chin and the welldefined eye brow. This taxon was not observed by A.P. Kinloch (1921b,c; 1923a,b) during his studies in Nelliampathies. The northern most range of this subspecies until now was considered as Palani Hills. Hence, the range extension of this species, though not unexpected, is of interest. Rasmussen \& Anderton (2005) have elevated it into a species endemic to Kerala called the Kerala Laughingthrush Trochalopteron fairbanki.

Broad-tailed Grass-Warbler (Schoenicola platyura): The species was neither observed during the pilot study nor during the intensive study phase probably due to its secretive habits during non-breeding season. However, J. Praveen observed five individuals flitting among the grass near Nellikkulam peak in May 2003. One individual was observed delivering a "lark-like" song while perched on a rock indicating the birds were possibly breeding in the neighborhood. This warbler was not recorded by A.P. Kinloch nor by any other person from this range and hence remains the only record for Nelliampathies.

Nilgiri Pipit (Anthus nilghiriensis): Recorded from the grassy peaks of Nellikkulam and Pothumala during the pilot survey. C. Sashikumar noted that the birds seen at Pothumala had nesting material in its beak and hence must possibly be breeding. A.P. Kinloch has seen this bird only in the "Lily downs of the Cochin Nelliampathies".

Brown Rock Pipit (Anthus similis travancoriensis): This is the first record of this species from Nelliampathies. Two birds were seen on a rocky barren hill on the northern tip of the range near Kaikatty by J. Praveen \& Prasanth Narayanan. Subspecies identification is uncertain as no specimen capture was done but the birds were similar to the ones seen in Ashambu Hills. As Nelliampathies lies south of Palghat gap, it is expected that the subspecies found here would be travancoriensis. Hence, it is a range extension for this subspecies which is known only up to Palani Hills, south of Palghat gap (Ali \& Ripley, 1984).

Oriental Tree Pipit (Anthus hodgsoni): One bird seen at Karimala on 29 December 2003 at Karimala by Dipu Karuthedathu and P.P. Sreenivasan. A rare winter visitor to Kerala. 
Table 4. Birds recorded in earlier studies, but not recorded from Nelliampathies in the present study

\begin{tabular}{ll}
\hline & Species \\
\hline 1. & Indian White-backed Vulture (Gyps bengalensis) \\
2. & Oriental Hobby (Falco severus) \\
3. & Common Greenshank (Tringa nebularia) \\
4. & Eurasian Woodcock (Scolopax rusticola) \\
5. & Yellow-legged Green-Pigeon (Treron phoenicoptera) \\
6. & Green Imperial-Pigeon (Ducula aenea) \\
7. & Brown Wood-Owl (Strix leptogrammica) \\
8. & Short-eared Owl (Asio flammeus) \\
9. & Oriental Bay-Owl (Phodilus badius ripleyi) \\
10. & Malabar Lark (Galerida malabarica) \\
11. & Striated Swallow (Hirundo striolata) \\
12. & Rufous-backed Shrike (Lanius schach) \\
13. & Blue Rock-Thrush (Monticola solitarius) \\
14. & Indian Blue Robin (Luscinia brunnea) \\
15. & Pied Thrush (Zoothera wardii) \\
16. & Scaly Thrush (Zoothera dauma) \\
\hline
\end{tabular}

Birds that were not recorded from Nellimapathies during the present study are listed in Table 4. These birds recorded by A.P. Kinloch or other workers could not be located during the present study. At least some of these birds could have become 'locally extinct' from Nelliampathies.

It is also worth mentioning that one of the landmark discoveries in the ornithology of Western Ghats, the discovery of a new subspecies of Oriental Bay Owl (Phodilus badius ripleyi) was made from Periashola in the southern slopes of Nelliampathies (Hussain \& Khan, 1977). Rasmussen \& Anderton (2005) place this subspecies as endemic species to southern Western Ghats and Sri Lanka calling it Ceylon Bay Owl Phodilus assimilis. However, we did not come across this bird though we tried playback of tape-recorded calls in 'ideal' habitats. The Striated Swallow Hirundo striolata record by Kinloch $(1921 \mathrm{c})$ is a likely misidentification as the species is known to occur only in northeastern India. It is likely that the taxon he referred to was one of the migrant races of Redrumped Swallow Hirundo daurica.

\section{Conclusion}

Nelliampathies, taking into account its ornithological significance, has been identified as one of the Important Bird Areas of India (Islam \& Rahmani, 2003). The remaining natural patches of Nelliampathies, should be added to the adjoining Parambikulam Wildlife Sanctuary and Chalakudy reserve forests, together which could make it into a biodiversity zone, exclusively for tropical biodiversity research.

\section{ReFERENCES}

Ali, S. \& D.S. Ripley (1987). Compact Handbook of Birds of India and Pakistan. $2^{\text {nd }}$ Edition. Oxford University Press, 737pp.

Birdlife International (2001). Threatened Birds of Asia. Birdlife International Red Data Book, Cambridge, U.K., 2985pp.

Daniels, R.J.R. (1989). A Conservation Strategy for the birds of the Uttara Kannada district, Ph.D. thesis, Indian Institute of Science, Bangalore, 298pp.

Daniels, R.J.R., M. Hedge \& M. Gadgil (1990). Birds of man-made ecosystems: the plantations. Proceedings of Indian Academy of Sciences (Animal Science) 99(1): 79-89.

Grimmett, R., C. Inskipp \& T. Inskipp (1998). Birds of the Indian Subcontinent. Oxford University Press. Delhi, 888pp.

Hussain, S.A. \& M.A.R. Khan (1977). A new subspecies of Bay owl (Phodilus badius) (Horsfield) from peninsular India. Journal of the Bombay Natural History Society 74(2): 334-336.

Inskipp, T., N. Lindsey \& W.A. Duckworth (Eds.) (1996). An Annotated Checklist of the Birds of the Oriental Region. Oriental Bird Club, Bedfordshire, U.K., 294pp. Islam, M.Z. \& A.R. Rahmani (2004). Important Bird Areas of India. Priority Sites for Conservation. Indian Bird Conservation Network: Bombay natural History Society and Birdlife International, U.K., xviii + 1133pp.

Kazmierczak, K. (2000). A Field Guide to the Birds of India. Helm Information Ltd., U.K., 352pp.

Khan, M.A.R. (1977). Ecology and Behaviour of the Black-and-Orange Flycatcher Muscicapa nigrorufa (Jerdon). Ph.D. Thesis, University of Bombay, Bombay, India.

Kinloch, A.P. (1921a). Occurrence of the Pied Ground Thrush (Geocichla wardi) on the Nelliampathy Hills. Journal of the Bombay Natural History Society 27(4): 944 .

Kinloch, A.P. (1921b). Rough notes on the avifauna of the Nelliampathy Hills. Journal of the Bombay Natural History Society 27(4): 939-944.

Kinloch, A.P. (1921c). The avifauna of the Nelliampathy Hills. Journal of the Bombay Natural History Society 28(1): 279-280.

Kinloch, A.P. (1923a). Further notes on the avifauna of the Nelliampathy Hills. Journal of the Bombay Natural History Society 29(1): 294.

Kinloch, A.P. (1923b). On the birds of the Nelliampathy Hills. Journal of the Bombay Natural History Society 29(2): 564-565.

Manakadan, R \& A. Pittie (2001). Standardised common and scientific names of the birds of the Indian subcontinent. Buceros 6(1): 1-38.

Nair, S.C. (1991). The Southern Western Ghats - A Biodiversity Conservation Plan. Intach, New Delhi, 92pp.

Prasad, S.N. \& L. Vijayan (2002). Conservation Planning for the Western Ghats of Kerala, pp.99-132. In: Biodiversity Hotspots Conservation Programme (BHCP), Final Report 1992-2002, Vol-I (Western Ghats). Forest and Biodiversity Conservation Division, world Wide Fund for Nature-India, New Delhi. Pp. 195.

Rasmussen, P.C. \& J.C. Anderton (2005). Birds of South Asia - The Ripley Guide. Volume 1: Field Guide. Smithsonian Institution \& Lynx Edicions, Washington DC, 378pp.

Stattersfield, A.J., M.J. Crosby, A.J. Long \& D.C. Wege (1998). Endemic Bird Areas of the World, Priorities for Biodiversity Conservation. BirdLife International, Cambridge.

Stotz, D.F., J.W. Fitzpatrick, T.A. Parker \& D.K. Moskovits (1996). Neotropical Birds - Ecology and Conservation. University of Chicago Press, Chicago.

Vijayan, V.S. (1978). Parambikulam Wildlife Sanctuary and its adjacent areas. Journal of the Bombay Natural History Society 75(3): 888-900.

Zacharias, V.J. \& A.J. Gaston (1999). The recent distribution of endemic, disjunct and globally uncommon birds in the forests of Kerala, State, south-west India. Bird Conservation International 9: 191-225.

\section{ACKNOWLedgements}

We would like to acknowledge the Oriental Bird Club, UK for sanctioning the project under the "OBC Small (Conservation) Grant. OBC through their "Small (Conservation) Grant" programme has been contributing substantially to the improvement of knowledge of avifauna of the Oriental region. We thankfully acknowledge the support extended by the officers of the Kerala State Forest Department, Kerala Forest Development Corporation and Kerala State Electricity Board, former for granting permission of the study and latter for the logistic support. Many thanks to all the participants of the pilot survey and the intensive survey for their many hours in the field without which we would not have been able to generate this data. We would like to specially thank L. Namassivayan, C. Sashikumar, Jafer Palot, K.G. Raghu, E. Kunhikrishnan, Sathyan Meppyaur, P.K. Ravindran and P.P. Sreenivasan for having lead individual teams on different transects, contributing extensively in preparing an authentic checklist after the survey and reviewing earlier drafts of this manuscript. We also thank the unknown reviewers for offering very useful comments on the earlier draft of the manuscript and there by helping to improve the quality of the paper. The second author would like to thank the authorities of Kerala Agricultural University and College of Forestry for their support.

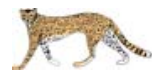

